\title{
Brain Metastasis From Papillary Thyroid Carcinoma in a Seventy-Three-Year- Old Man
}

\author{
Mohammad Reza Emamhadi ${ }^{1}$; Babak Alijani ${ }^{1, *}$; Shahrokh Yousefzadeh Chabok ${ }^{1}$; Hamid \\ Behzadnia ${ }^{1}$; Siavash Dehghani ${ }^{1}$ \\ ${ }^{1}$ Department of Neurosurgery, Poursina Hospital, Guilan University of Medical Sciences, Rasht, IR Iran \\ *Corresponding author: Babak Alijani, Department of Neurosurgery, Poursina Hospital, Guilan University of Medical Sciences, Rasht, IR Iran. Tel: +98-1313227346, E-mail:drbabakalij@ \\ gmail.com \\ Received: April 21, 2014; Revised: August 24, 2014; Accepted: November 15, 2014
}

\begin{abstract}
Introduction: Papillary carcinoma of the thyroid is the most common differentiated malignancy of thyroid, which is characterized by slow progression and a good prognosis. Being extremely rare, brain metastases from papillary thyroid carcinoma (PTC) have a poor prognosis; they are usually asymptomatic, but a few could be very symptomatic and life threatening.

Case Presentation: We report a rare case of brain metastasis from PTC with sudden loss of consciousness and normal follow-up screening tests, 17 years after primary diagnosis of PTC. The patient underwent radical surgery urgently and the histopathologic diagnosis of metastatic brain tumor of thyroid papillary carcinoma was made. Postoperative course was uneventful, and the patient's neurologic deficits improved.

Discussion: An early diagnosis and treatment of brain metastasis of PTC can result in longer survival time for patients and magnetic resonance imaging can be used as the most sensitive method for detecting brain metastases. The treatment modalities must be individualized and surgery could be a more suitable treatment option in case of hemorrhagic metastasis.
\end{abstract}

Keywords:Brain; Metastasis; Thyroid Carcinoma

\section{Introduction}

Papillary thyroid carcinoma (PTC) is the most common thyroid malignancy, accounting for $70 \%$ to $80 \%$ of all thyroid malignancies. It typically occurs in the middle age with a peak incidence in the third and fourth decades. It is the predominant thyroid cancer in children and individuals exposed to external radiations. In comparison to other differentiated and undifferentiated thyroid malignancies, PTC is characterized by an indolent clinical course. Metastases are uncommon and usually involve regional lymph nodes. The distant metastases, mainly including lungs and bone lesions, are very uncommon. They are detected in $6 \%$ to $20 \%$ of patients during followup (1-3). Brain metastases, with about $1 \%$ incidence, suggest an aggressive disease and specifically have unfavorable prognosis of less than a year survival. Hence, early detection and appropriate treatment of brain metastasis is necessary, because it can lead to long-term survival (410). We report a rare case of brain metastasis from PTC in a 73-year-old man who presented with sudden loss of consciousness and normal findings on follow-up screening tests, 17 years after primary diagnosis of PTC.

\section{Case Presentation}

A 73-year-old man presented with sudden loss of con- sciousness (Glasgow coma scale [GCS], 7), left hemiparesis and anisocoria. He was a known case of PTC diagnosed 17 years before presentation, which had led to a total thyroidectomy. The patient had two neck dissections because of lymph nodes metastases and four courses of cervical iodine treatments, the last of which was 13 months ago. Whole body scan by $\mathrm{I}^{131}$ demonstrated no evidence for residual active thyroid tissue or metastatic disease after the last iodine treatment. Results of recent serum thyroglobulin, thyroglobulin antibody, and thyroid function tests were in the normal ranges.

Brain computed tomography (CT) demonstrated a hemorrhagic mass lesion in the left frontal lobe with considerable midline shift (Figure 1). Based on signs of progressive neurologic deterioration referable to the lesion, medically refractory intracranial hypertension, and signs of a mass effect on CT, we decided to operate the patient urgently. A unilateral frontal craniotomy through a curved skin incision was performed and the lesion was addressed via transcortical approach. The hemorrhagic and well-circumscribed lesion was readily separated from cerebral parenchyma and the lesion was totally resected macroscopically. Tissue histopathologic examination revealed metastatic brain tumor of PTC origin, char- 
acterized by composition of papillary structures, lined by neoplastic cells with overlapping nuclei (Figure 2). Immunohistochemical analysis confirmed the accuracy of diagnosis (strongly positive reaction to thyroglobulin and thyroid transcription factor 1 [TTF1]) (Figures 3 and 4). Postoperative course was uneventful, and the hemiparesis and loss of consciousness improved.

\section{Discussion}

PCT is a well-differentiated thyroid carcinoma and a relatively uncommon malignancy that might metastasize. Metastatic lesions are usually seen in the regional lymph nodes and the most common site of distant metastases is the lung, followed by the bone. However, brain metastases are extremely unusual with an incidence rate of $0.15 \%$ to $1.3 \%$. In the case of brain metastasis by PTC, cerebral hemispheres are the common site of involvement followed by cerebellum and pituitary gland. Brain metastases are usually asymptomatic, and only a few have suggestive symptoms, including headache, visual disturbances, or ocular motor weakness (1-6). Acute and severe spontaneous cerebral hemorrhage is a life threatening complication that can lead to abrupt onset of neurologic symptoms and rapid death due to intratumoral hemorrhage. Hemorrhagic intracranial metastases represent 3\% to 14\% of all cerebral metastases and they classically originate from renal cell carcinoma, melanoma, choriocarcinoma, bronchogenic carcinoma, and hepatocellular carcinoma (11-13). The presented case and sporadic cases of hemorrhagic brain metastases caused by PTC, demonstrate that PTC should be considered in the differential diagnosis of hemorrhagic intracranial metastases (11, 13-15).

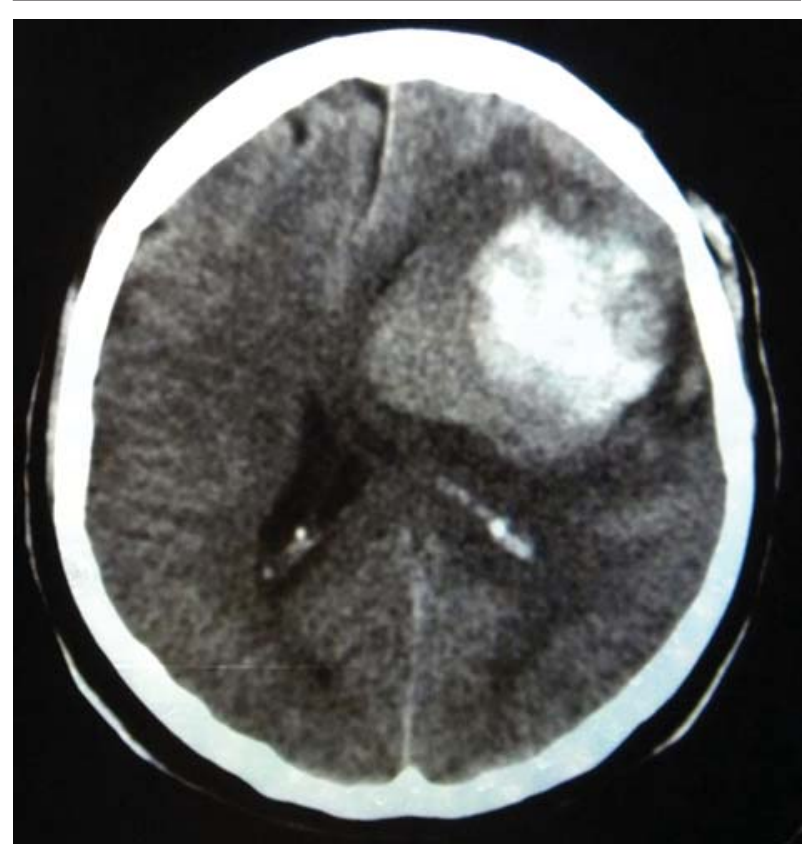

Figure 1. Patient's Brain Computed Tomographic Scan Shows a Hemorrhagic Mass Lesion in the Left Frontal Lobe

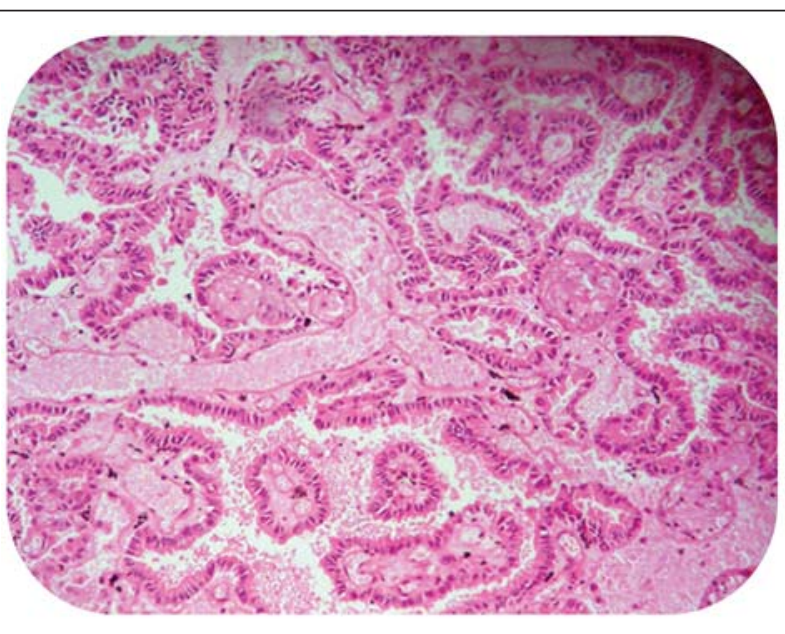

Figure 2. The Tumor Composed of Papillary Structures, Lined by Neoplastic Cells With Overlapping Nuclei (Hematoxylin and Eosin stain, 100X magnification)

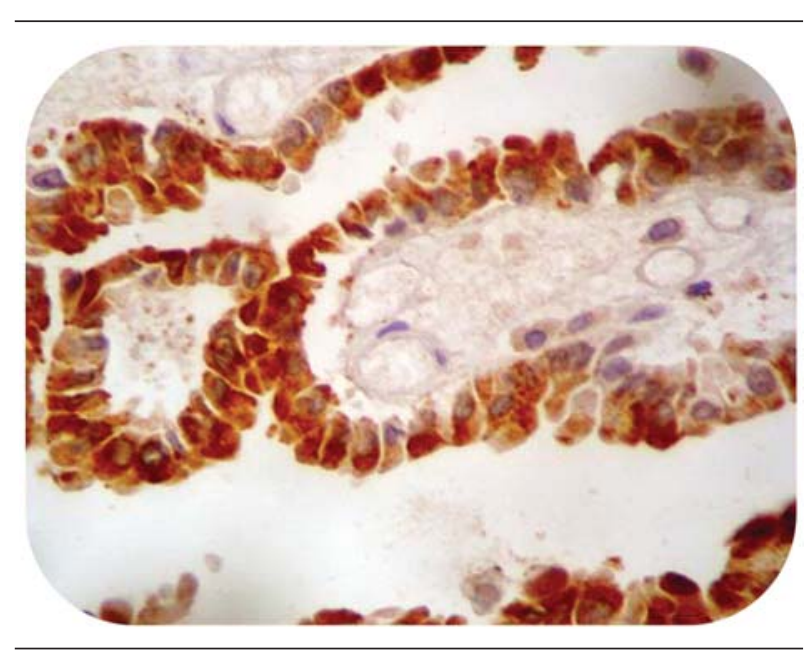

Figure 3. The Tumoral Cells With Strong Positive Reaction to Thyroglobulin (Immunohistochemical stain)

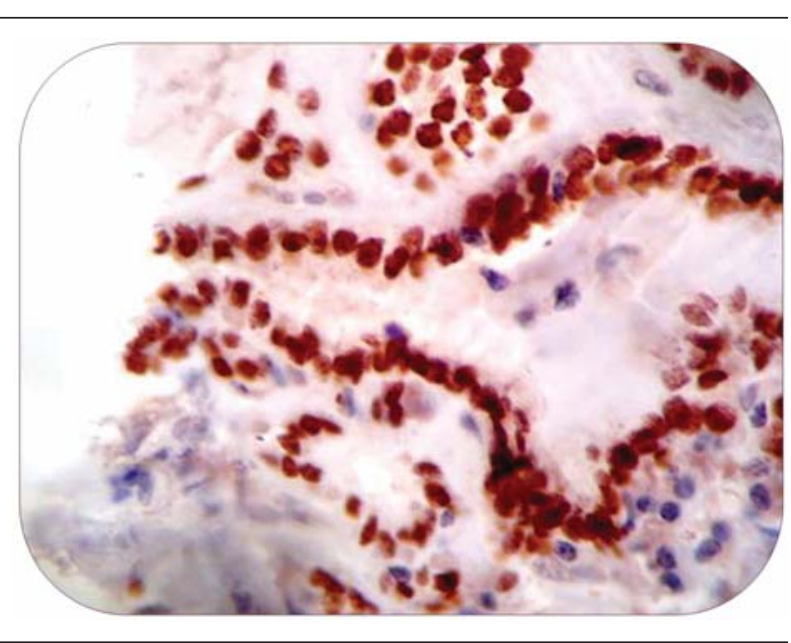

Figure 4. The Tumoral Cells With Strong Positive Reaction to Thyroid Transcription Factor 1TTF1 (Immunohistochemical Stain) 
Some risk factors for distant metastasis of PTC include male sex, advanced age, histologic grade, extrathyroidal invasion at initial examination, and multiple organs involvement $(16,17)$. In general, patients with PTC have an excellent prognosis, with a 10-year survival rate of more than 95\%. Several prognostic indicators have been incorporated into various staging systems, including AGES scoring system, MACIS scale and TNM system. Patients with brain metastases are placed in high-risk groups in all staging systems and have an unfavorable prognosis of less than one year $(18,19)$. PTC has a relatively benign course, and early detection and surgical removal of brain metastasis prolong patients' survival. Except for the information obtained from the patient's history, physical examination, and diagnostic studies, there are no definitive methods for diagnosing metastases of this carcinoma to the brain. Only histologic examination provides confirmation of the diagnosis. Screening with $\mathrm{I}^{131}$ is more sensitive than chest X-ray or CT scanning in detecting metastases; however, it is less sensitive than thyroglobulin measurements in detecting metastatic diseases in most differentiated thyroid cancers, except in Hurtle cell tumors (20). Metastatic lesions from differentiated thyroid carcinomas are usually less differentiated than primary tumors. Some metastatic lesions, especially brain metastases, do not have differentiation, leading to no accumulation of $\mathrm{I}^{131}$ in the metastatic sites (21). Salvati et al. reported 12 patients with single brain metastases and none of them had positive findings in $\mathrm{I}^{131}$ whole body scan (22). Fluorine-18-FDG-PET/CT is a sensitive method to detect metastases, which reveal negative results for $\mathrm{I}^{131}$ whole body scan in patients with elevated levels of serum thyroglobulin. However, compared with MRI, Fluorine18-FDG-PET/CT cannot reveal all brain metastatic lesions; Therefore, magnetic resonance imaging (MRI) is the most sensitive method for detection of brain metastasis (23). Optimal treatment protocol for patients with intracranial metastatic tumors of PTC origin is not clearly defined, and treatment should be tailored to each patient individually. Several treatment modalities with varying results, e.g. surgical resection, gamma knife radiosurgery, whole brain radiation therapy, radioiodine therapy, and chemotherapy, have been used in the limited number of intracranial metastatic PTCs (13, 24-27). Although the presence of a brain metastasis is an overall negative prognostic indicator, surgical resection of brain metastases might help to significantly prolong survival in patients with differentiated thyroid carcinoma, especially in solitary or oligometastatic lesions (13, 24-26). In life-threatening complications such as refractory intracranial hypertension, severe brain edema, tonsillar herniation, and intracranial hemorrhage, surgery is also the treatment of choice $(13,24)$. However, if a patient is at high risk for surgery or surgery is impossible and in the case of incomplete resection of metastatic lesions or diagnostic biopsy, adjuvant therapy such as whole brain radiotherapy or radiosurgery might be the best therapeutic choice. Whole brain radiation external beam therapy is usually used to treat multiple lesions. There are very few data regarding the efficacy of whole brain radiation therapy after surgery and long-term complications must be considered in patients who are likely to have prolonged survival $(24,27$, 28). Gamma knife radiosurgery certainly is an attractive option for the local control of a solitary lesions or a few smaller ones because it is less invasive than craniotomy; it is especially important in patients who might have a limited survival time (24). Patients treated with SRS (stereotactic radio surgery) for brain metastases from primary thyroid cancer have a favorable prognosis with an expected median survival of longer than three years $(13,29)$. Radioiodine treatment would be a reasonable treatment option if findings of the $\mathrm{I}^{131}$ whole body scan were positive. There have been cases in which a response to such treatment was achieved $(24,27)$. Chemotherapy has only a limited role in the management of thyroid carcinoma with extracranial metastases $(23,24)$, and this appears to hold true for intracranial metastases as well (24). Miranda et al. reported a case with ten-year survival (the longest survival of a patient with brain metastases by far), who was managed by combined approach of surgical excision, $\mathrm{I}^{131}$, whole-brain radiation therapy, and gamma knife radiosurgery (30).

Based on a summary of findings from previous case reports, the present case showed some remarkable characteristics. First, the patient presented with acute neurologic deficits due to intracerebral hemorrhage and no other sites of distant tumor dissemination were detected (9, $11,31)$. Therefore, surgery could be a more suitable treatment option in case of hemorrhagic metastasis because surgery can relieve neurologic deficits immediately (32). Second, $\mathrm{I}^{131}$ whole body scan demonstrated no evidence of residual active thyroid tissue or metastatic disease, while results of serum thyroglobulin and thyroglobulin antibody were in the normal ranges $(1.6 \mathrm{ng} / \mathrm{mL}$ and $16.6 \mathrm{IU} / \mathrm{mL}$, respectively). Therefore, we recommend MRI as the most sensitive method for the detection of brain metastases as reported by Xu et al. (23), and it should be taken into consideration in patients with presentation suggestive of brain metastasis, even with normal serum thyroglobulin levels. Third, a combined approach of treatment modalities must be individually modified for each patient.

\section{Acknowledgements}

We acknowledge the pathology center of Rasht Poursina Hospital for preparing the specimens.

\section{References}

1. Sugitani I, Fujimoto Y, Yamamoto N. Papillary thyroid carcinoma with distant metastases: survival predictors and the importance of local control. Surgery. 2008;143(1):35-42.

2. Haq M, Harmer C. Differentiated thyroid carcinoma with distant metastases at presentation: prognostic factors and outcome. Clin Endocrinol (Oxf). 2005;63(1):87-93. 
3. Mihailovic J, Stefanovic L, Malesevic M. Differentiated thyroid carcinoma with distant metastases: probability of survival and its predicting factors. Cancer Biother Radiopharm. 2007; 22(2):250-5.

4. Aguiar PH, Agner C, Tavares FR, Yamaguchi N. Unusual brain metastases from papillary thyroid carcinoma: case report. Neurosurgery. 2001;49(4):1008-13.

5. Hoie J, Stenwig AE, Kullmann G, Lindegaard M. Distant metastases in papillary thyroid cancer. A review of 91 patients. Cancer. 1988;61(1):1-6.

6. Biswal BM, Bal CS, Sandhu MS, Padhy AK, Rath GK. Management of intracranial metastases of differentiated carcinoma of thyroid. J Neurooncol. 1994;22(1):77-81.

7. Aihara N, Nagai H, Mase M, Shimazu N, Kanai H, Kamiya K. Brain metastasis of thyroid papillary carcinoma--case report. Neurol Med Chir (Tokyo). 1991;31(6):356-8.

8. Dinneen SF, Valimaki MJ, Bergstralh EJ, Goellner JR, Gorman CA Hay ID. Distant metastases in papillary thyroid carcinoma: 100 cases observed at one institution during 5 decades. J Clin Endocrinol Metab. 1995;80(7):2041-5.

9. Ota T, Bando Y, Hirai M, Tanaka N, Takabatake Y, Kasahara Y, et al. Papillary carcinoma of the thyroid with distant metastases to the cerebrum: a case report.Jpn J Clin Oncol. 2001;31(3):112-5.

10. Hay ID, Grant CS, Taylor WF, McConahey WM. Ipsilateral lobectomy versus bilateral lobar resection in papillary thyroid carcinoma: a retrospective analysis of surgical outcome using a novel prognostic scoring system. Surgery. 1987;102(6):1088-95.

11. Chonan M, Mino M, Yoshida M, Sakamoto K. [Brain metastasis from papillary thyroid carcinoma with acute intracerebral hemorrhage: a surgical case report]. No Shinkei Geka.2012;40(5):453-7.

12. Lieu AS, Hwang SL, Howng SL, Chai CY. Brain tumors with hemorrhage. Formos J Med Assoc. 1999;98(5):365.

13. Tanaka T, Kato N, Aoki K, Nakamura A, Watanabe M, Arai T, et al Cerebellar hemorrhage secondary to cerebellopontine angle metastasis from thyroid papillary carcinoma. Neurol Med Chir (Tokyo). 2013;53(4):233-6.

14. Lin CK, Lieu AS, Howng SL. Hemorrhagic cerebellar metastasis from papillary thyroid carcinoma. Kaohsiung J Med Sci. 1999;15(4):234-8.

15. Lecumberri B, Alvarez-Escola C, Martin-Vaquero P, Nistal M, Martin V, Riesco-Eizaguirre G, et al. Solitary hemorrhagic cerebellar metastasis from occult papillary thyroid microcarcinoma. Thy roid. 2010;20(5):563-7.

16. Song HJ, Xue YL, Xu YH, Qiu ZL, Luo QY. Rare metastases of differentiated thyroid carcinoma: pictorial review. Endocr Relat Cancer 2011;18(5):R165-74

17. Cha ST, Jarrahy R, Mathiesen RA, Suh R, Shahinian HK. Cerebellopontine angle metastasis from papillary carcinoma of the thyroid: case report and literature review. Surg Neurol.
2000;54(4):320-6.

18. Cady B, Rossi R. An expanded view of risk-group definition in differentiated thyroid carcinoma. Surgery. 1988;104(6):947-53.

19. Tahmasebi FC, Farmer P, Powell SZ, Aldape KD, Fuller GN, Patel S et al. Brain metastases from papillary thyroid carcinomas. Virchows Arch. 2013;462(4):473-80.

20. Cooper DS, Doherty GM, Haugen BR, Kloos RT, Lee SL, Mandel SJ, et al. Management guidelines for patients with thyroid nodules and differentiated thyroid cancer. Thyroid. 2006;16(2):109-42.

21. Parker LN, Wu SY, Kim DD, Kollin J, Prasasvinichai S. Recurrence of papillary thyroid carcinoma presenting as a focal neurologic deficit. Arch Intern Med.1986;146(10):1985-7.

22. Salvati M, Frati A, Rocchi G, Masciangelo R, Antonaci A, Gagliardi FM, et al. Single brain metastasis from thyroid cancer: report of twelve cases and review of the literature. J Neurooncol. 2001;51(1):33-40.

23. Xu YH, Song HJ, Qiu ZL, Luo QY. Brain metastases with exceptional features from papillary thyroid carcinoma: report of three cases. Hell J Nucl Med. 2011;14(1):56-9.

24. McWilliams RR, Giannini C, Hay ID, Atkinson JL, Stafford SL, Buckner JC. Management of brain metastases from thyroid carcinoma: a study of 16 pathologically confirmed cases over 25 years. Cancer. 2003;98(2):356-62.

25. Tsuda K, Tsurushima H, Takano S, Tsuboi K, Matsumura A. Brain metastasis from papillary thyroid carcinomas. Mol Clin Oncol. 2013;1(5):817-9.

26. Wasita B, Sakamoto M, Mizushima M, Kurosaki M, Watanabe T. Choroid plexus metastasis from papillary thyroid carcinoma presenting with intraventricular hemorrhage: case report. Neurosurgery. 2010;66(6):E1213-4.

27. Vrachimis A, Schmid KW, Jurgens H, Schober O, Weckesser M Riemann B. Cerebral metastases from thyroid carcinoma: complete remission following radioiodine treatment. Dtsch Arztebl Int. 2013;110(50):861-6.

28. Chiu AC, Delpassand ES, Sherman SI. Prognosis and treatment of brain metastases in thyroid carcinoma. J Clin Endocrinol Metab. 1997;82(11):3637-42.

29. Bernad DM, Sperduto PW, Souhami L, Jensen AW, Roberge D. Stereotactic radiosurgery in the management of brain metastases from primary thyroid cancers. J Neurooncol. 2010;98(2):249-52.

30. Miranda ER, Padrao EL, Silva BC, De Marco L, Sarquis MS. Papillary thyroid carcinoma with brain metastases: an unusual 10-yearsurvival case. Thyroid. 2010;20(6):657-61.

31. Jyothirmayi R, Edison J, Nayar PP, Nair MK, Rajan B. Case report: brain metastases from papillary carcinoma thyroid. Br J Radiol. 1995;68(811):767-9.

32. Yoo H, Jung E, Gwak HS, Shin SH, Lee SH. Surgical outcomes of hemorrhagic metastatic brain tumors. Cancer Res Treat. 2011;43(2):102-7. 